\title{
Multifocus self-focusing of a femtosecond optical vortex
}

\author{
Evgeny Vasilyev*, Svyatoslav Shlenov, and Valery Kandidov
}

Lomonosov Moscow State University, Faculty of Physics and International Laser Center, 119991, Moscow, Russia

\begin{abstract}
We analyze formation of multifocus structure during the propagation of optical vortex with topological charge $m=1$ in fused silica at wavelength $1800 \mathrm{~nm}$, which corresponds to region of anomalous group velocity dispersion. Propagation of pulse is accompanied by sequence of nonlinear focuses, where each focus is a high intensive ring.
\end{abstract}

Self-action of femtosecond pulses may tend to formation of spatially long structures with high intensity - filaments [1]. Propagation of laser pulses in the regime of anomalous group velocity dispersion (GVD) may be accompanied by formation of localized in space and time high intensive structures - "light bullets" [2, 3]. Filamentation in beams with smooth phase has been widely studied for Gaussian beams, Airy and Bessel beams [4]. Annular beams with spiral phase dislocation have zero field on optical axis and are often called optical vortices. Self-focusing of optical vortex in a medium with normal GVD was studied in $[5,6]$. Filamentation of vortices often tends to breaking up into several hot points due to modulational instability [7], but the distance of nonlinear focus can be less than the distance of modulational hot points formation. Thus, nonlinear focus preserves annular symmetry.

The formation of tubular filament with peak intensity $5 \times 10^{13} \mathrm{~W} / \mathrm{cm}^{2}$ and plasma electrons concentration $10^{-3} N_{0}$ of neutrals, was demonstrated in [9] for femtosecond optical vortex in fused silica with normal GVD at central wavelength $800 \mathrm{~nm}$. This work is devoted to numerical simulations of optical vortex filamentation in the presence of anomalous GVD. We analyze dynamics of light bullets and evolution of fluence distribution of the pulse.

Numerical simulation of the optical vortex self-action is based on the system of equations for slowly varying complex amplitude of laser field $A_{V}(r, \varphi, t, z)=A(r, t, z) e^{i m \varphi}$ and free electron concentration $N_{e}(r, t)$ :

$$
\left\{\begin{aligned}
2 i k_{0} \frac{\partial A}{\partial z}=\widehat{T}^{-1}\left(\frac{\partial^{2}}{\partial r^{2}}+\frac{1}{r} \frac{\partial}{\partial r}\right. & \left.-\frac{m^{2}}{r^{2}}\right) A+\widehat{T}^{-1} \widehat{D} A+\frac{2 k_{0}^{2}}{n_{0}} \widehat{T} \Delta n_{k} A-\frac{2 k_{0}^{2}}{n_{0}} \widehat{T}^{-1} \Delta n_{p l} A+ \\
& +i \widehat{T}^{-2} \sigma A-i k_{0}(\alpha+\delta) A \\
\frac{\partial N_{e}}{\partial t} & =W\left(N_{0}-N_{e}\right)+N_{e}\left(v_{i}-\beta\right)
\end{aligned}\right.
$$

Operator of the wave nonstationarity $\hat{T}$ [9] is used to describe such effects as femtosecond pulse wavefront self-steepening.

\footnotetext{
*Corresponding author: vasilev.evgeniy@physics.msu.ru
} 
Initial shape of optical vortex was considered as annular vortex beam with Gaussian envelope:

$$
A_{V}(r, \varphi, t, z=0)=A_{0}\left(\frac{r}{r_{0}}\right)^{m} e^{-r^{2} / 2 r_{0}} e^{-t^{2} / 2 t_{0}} e^{i m \varphi},
$$

where $m=1$ is topological charge, $\varphi=\arctan x / y$. Spatial parameter $r_{0}=100 \mu \mathrm{m}$, pulse duration $t_{0}=36 \mathrm{fs}$, central wavelength $\lambda_{0}=1800 \mathrm{~nm}$. Peak power of initial beam was $P_{0}=$ $5 P_{V}$, where $P_{V}$ is the critical power of self-focusing for optical vortex [5,6] with topological charge $m=1$.

In the initial stage of vortex beam propagation the self-action yields narrowing ring in the beam cross-section, its radius being practically the same $r_{0}=100 \mu \mathrm{m}$ at $z=0.9 \mathrm{~cm}$ (Fig. 1). Peak intensity rises up to $1.5 \times 10^{13} \mathrm{~W} / \mathrm{cm}^{2}$, ring width decreases in $\sim 10$ times, peak fluence is $0.07 \mathrm{~J} / \mathrm{cm}^{2}$. This wave packet corresponds to the first nonlinear focus and can be considered as vortex bullet. Kerr's self-focusing is then stopped by linear diffraction, which is accompanied by flowing pulse energy towards the optical axis. Phase singularity prevents the appearance of the light field in the beam center and interference rings are formed at $z \sim 1.2-2.5 \mathrm{~cm}$ around the optical axis with fluence up to $0.04 \mathrm{~J} / \mathrm{cm}^{2}$. The next nonlinear focus takes place at distance $z=4.1 \mathrm{~cm}$. The light bullet corresponding to the second focus has approximately the same peak intensity as in the first focus, ring radius being about $50 \mu \mathrm{m}$. Energy flowing towards beam axis remains up to the distance of $z \sim 4.3 \mathrm{~cm}$, where the last focus is situated. Peak intensity reaches the global maximum, which is about $5 \times 10^{13}$ $\mathrm{W} / \mathrm{cm}^{2}$, fluence is $0.24 \mathrm{~J} / \mathrm{cm}^{2}$, the ring radius decreases up to $10 \mu \mathrm{m}$. Plasma electrons concentration is reached for the first time noticeable value $0.5 \times 10^{-3} N_{0}$, where $N_{0}$ is neutrals concentration.

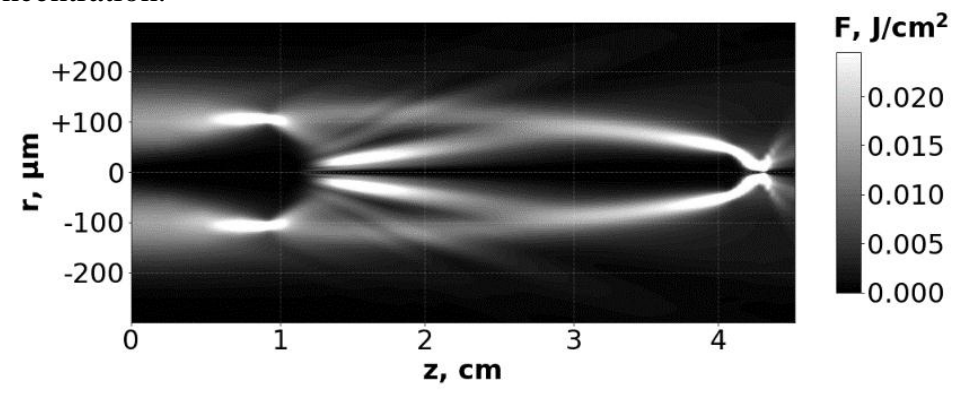

Fig. 1. Fluence evolution of optical vortex in fused silica at wavelength $1800 \mathrm{~nm}$.

This research was supported by the RFBR grant 18-02-00624.

\section{References}

1. V. P. Kandidov, S. A. Shlenov et al. Quant. El., 39, 205 (2009)

2. $\quad$ E. O. Smetanina, A.E. Dormidonov et al., Laser Phys., 22, 1189 (2012)

3. S. V. Chekalin, A. E. Dokukina et al., J. Phys. B, 48, 094008 (2015)

4. A. Couairon, A. Lotti et al., Proc. of SPIE, 8770, 87701E (2013)

5. V. I. Kruglov, Yu. A. Logvin et al., J. Modern Optics, 39, 2277 (1992)

6. L. T. Vuong, T. D. Grow et al., Phys. Rev. Let., 96, 133901 (2006)

7. M. S. Bigelow, P. Zerom, R.W. Boyd, Phys. Rev. Lett., 92, 083902 (2004)

8. R. A. Vlasov, V. M. Volkov, D. Yu. Dedkov, Quant. El., 43, 157 (2013)

9. E. V. Vasil'ev, S. A. Shlenov, Quant. El., 46, 1002 (2016) 